\title{
gs \\ Optical plasma torch electron bunch generation in plasma wakefield accelerators
}

\author{
G. Wittig, ${ }^{1}$ O. Karger, ${ }^{1}$ A. Knetsch, ${ }^{1}$ Y. Xi, ${ }^{2}$ A. Deng, ${ }^{2}$ J. B. Rosenzweig, ${ }^{2}$ D. L. Bruhwiler, ${ }^{3,4}$ \\ J. Smith, ${ }^{5}$ G. G. Manahan, ${ }^{6}$ Z.-M. Sheng, ${ }^{6}$ D. A. Jaroszynski, ${ }^{6}$ and B. Hidding ${ }^{1,2,6}$ \\ ${ }^{1}$ Institute of Experimental Physics, University of Hamburg, 22761 Hamburg, Germany \\ ${ }^{2}$ Particle Beam Physics Laboratory, UCLA, Los Angeles, California 90095, USA \\ ${ }^{3}$ RadiaSoft LLC, Boulder, Colorado 80304, USA \\ ${ }^{4}$ RadiaBeam Technologies LLC, Santa Monica, California 90404, USA \\ ${ }^{5}$ Tech-X UK Ltd, Daresbury, Cheshire WA4 4FS, United Kingdom \\ ${ }^{6}$ Physics Department, University of Strathclyde, Rottenrow, Glasgow G4 ONG, United Kingdom
}

(Received 27 February 2015; published 31 August 2015)

\begin{abstract}
A novel, flexible method of witness electron bunch generation in plasma wakefield accelerators is described. A quasistationary plasma region is ignited by a focused laser pulse prior to the arrival of the plasma wave. This localized, shapeable optical plasma torch causes a strong distortion of the plasma blowout during passage of the electron driver bunch, leading to collective alteration of plasma electron trajectories and to controlled injection. This optically steered injection is more flexible and faster when compared to hydrodynamically controlled gas density transition injection methods.
\end{abstract}

PACS numbers: 52.40.Mj, 29.27.Ac, 52.50.Dg, 52.65.Rr

The use of plasmas for acceleration of electrons is an increasingly vivid topic, fueled by the fundamental advantage that the extremely high electric fields available supersede those in conventional accelerators by many orders of magnitude. In recent years the ability to excite and control suitable plasma waves driven either by laser (laser wakefield acceleration, LWFA) [1-8] or electron beams (plasma wakefield acceleration, PWFA) [9-13] has increased substantially. In both cases, injection of electron beams into the proper phase of the plasma wave is of paramount importance to obtain high-quality witness bunches from the plasma. A multitude of injection methods has been conceived, among those hydrodynamics-based plasma density transition [14-20], injection by additional ionization [21-30] and Trojan Horse-type methods [31-38].

In this work, we present a novel method of generating sharp and easily tunable plasma electron density profiles, which can be used to produce high-quality electron bunches in beam-driven plasma waves, but also for example to shape plasma cell boundaries, and as an ultrafast electron bunch kicker [39]. This region, of optically enhanced and shaped plasma density, we refer to as "plasma torch." We discuss the experimental setup and explore and analyze the injection method for three different configurations, exemplified by simulations. The plasma density perturbation-plasma torch-is generated and controlled optically in a homogeneous gas reservoir, which offers many fundamental and

Published by the American Physical Society under the terms of the Creative Commons Attribution 3.0 License. Further distribution of this work must maintain attribution to the author(s) and the published article's title, journal citation, and DOI. practical advantages when compared to much slower hydrodynamics-based gas density perturbations. The technique is substantially different from a hybrid scheme discussed in [17,20,40], in the context of LWFA, where hydrodynamic expansion is required after optical excitation at near-relativistic intensities, such that a density transition arises on ns timescales due to ion motion. In contrast, here we create the plasma torch electron density elevation on fs to ps-timescale before arrival of the electron-beam driven plasma wave with Ti:sapphire laser pulses of intensities $I \approx 10^{15} \mathrm{~W} \mathrm{~cm}^{-2}$. This is enabled by the much lower electric fields of PWFA drivers when compared to LWFA drivers, which allows such comparably low laser intensities to tunnel ionize gas medium levels which would not be ionized by the electron bunch driver itself. For example, while in typical LWFA driver pulses with intensities of the order of $I \approx 10^{18} \mathrm{~W} \mathrm{~cm}^{-2}$ ionize each element of the periodic system, and in most cases even higher ionization threshold (HIT) levels, even low-ionization threshold (LIT) elements are hardly ionizable by the electric fields of typical PWFA electron bunch drivers.

We explore three fundamental cases of plasma torch electron bunch generation using a two component gas mixture with hydrogen as LIT and helium as HIT medium: (i) hydrogen and helium are present in the neutral state, the electron beam is capable to self-ionize hydrogen on axis, the plasma torch laser preionizes hydrogen locally—and no helium is ionized at all; (ii) same as case (i), but the torch laser ionizes both hydrogen and helium locally, (iii) hydrogen is completely preionized, and the torch laser ionizes additional helium locally. 
Experimental realization of such a setup for example in $90^{\circ}$ geometry is straightforward: In addition to a suitable electron driver bunch, one or two moderately (ps-level or better) synchronized laser pulses are needed with all three axes overlapping in a LIT/HIT mixture such as hydrogen/ helium. This is shown in Fig. 1. The plasma torch laser arm with a laser energy in the range of sub $\mathrm{mJ}$ to tens of $\mathrm{mJ}$ is essential for the scheme, and it needs to be focused by a lens or off-axis parabola (OAP) onto the electron beam axis. In case of a Ti:sapphire laser pulse with a central wavelength of $\lambda \approx 800 \mathrm{~nm}$, a peak intensity in the range of $I \approx 10^{14}-10^{15} \mathrm{~W} \mathrm{~cm}^{-2}$ is required to ionize either hydrogen [case (i)] or hydrogen and helium [case (ii)]. For case (iii), an additional laser arm is required which is needed to produce a preionized hydrogen plasma channel around the electron beam axis. This higher energy laser pulse needs softer focusing to produce a wide enough plasma channel to contain the blowout, which can be realized with a conventional lens, or parabolic mirror, or with an axicon (as sketched in Fig. 1) in order to realize a more effective laser intensity distribution and to avoid effects such as ionization defocusing.

The actual parameters for these three cases are chosen based on analytical estimations of suitable interaction processes, and are then examined using $3 \mathrm{~d}$ particle-in-cell (PIC) simulations with the code VSim/VORPAL [41]. For all cases simulations were made with and without the torch, showing clearly that trapping is a direct consequence of the plasma torch density perturbation.

The electron bunch driver is in all cases of FACET-class [13] with charge $Q=1-3 \mathrm{nC}$, energy $E=23 \mathrm{GeV}$, energy spread $\Delta E / E=2 \%$, bunch length $\sigma_{\mathrm{z}}=27 \mu \mathrm{m}$, diameter $\sigma_{\mathrm{r}}=8.5 \mu \mathrm{m}$, and normalized emittance $\epsilon_{\mathrm{n}}=2.25 \times 10^{-6} \mathrm{~m} \mathrm{rad}$. A laser pulse generates the plasma torch in perpendicular geometry approximately $1 \mathrm{ps}$ before

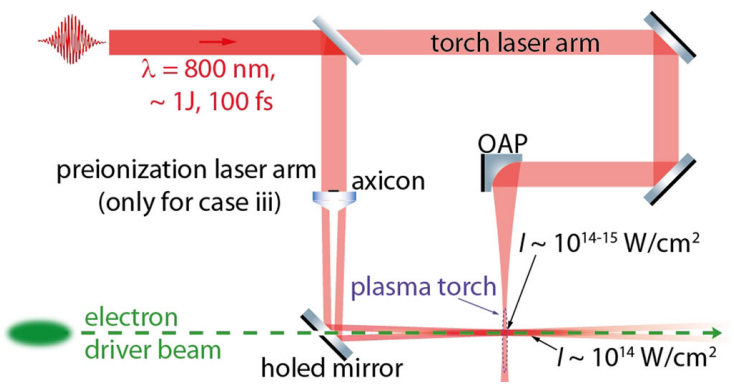

FIG. 1. Experimental setup: An electron beam driver and one or two moderately synchronized laser pulses interact in an underdense medium with (at least) two species with different ionization thresholds such as hydrogen and helium. One Ti:sapphire laser pulse is focused to intensities of the $I \approx 10^{14-15} \mathrm{~W} / \mathrm{cm}^{2}$ level in order to generate the localized hydrogen/helium plasma torch in the path of the electron beam driven blowout. In contrast to the self-ionized cases (i) and (ii), for case (iii) another, high-energy fraction of the laser pulse is used to preionize the hydrogen in order to allow for a stronger blowout. arrival of the electron-beam driven plasma wave, here we use a Ti:sapphire at $\lambda=800 \mathrm{~nm}$ wavelength, and $\tau=64 \mathrm{fs}$ (FWHM) duration. In the self-ionized cases (i) and (ii), the hydrogen and helium gas densities are set to $n_{\mathrm{H}}=n_{\mathrm{He}}=$ $5 \times 10^{17} \mathrm{~cm}^{-3}$ to ensure comparability, while in the preionized case (iii) the density is set to a substantially lower value of $n_{\mathrm{H}}=n_{\mathrm{He}}=1 \times 10^{16} \mathrm{~cm}^{-3}$ due to the much stronger blowout, and also the driver was set to a lower charge of $1 \mathrm{nC}$ compared to $3 \mathrm{nC}$ used in the first two cases.

The two ionization thresholds of hydrogen and helium require different laser intensities, which were set to $a_{0}=0.015$, corresponding to $I=a_{0}^{2} 2 \epsilon_{0} c\left[\pi m_{e} c^{2} /(e \lambda)\right]^{2} \approx$ $4.8 \times 10^{14} \mathrm{~W} \mathrm{~cm}^{-2}$ (with $\epsilon_{0}$ the vacuum permittivity, $m_{e}$ the electron mass, $c$ the vacuum speed of light, and $e$ the electron charge) for hydrogen-ionization in case (i), and $a_{0}=0.025$, corresponding to $I \approx 1.3 \times 10^{15} \mathrm{~W} \mathrm{~cm}^{-2}$ for helium-ionization as required in cases (ii) and (iii).

Figure 2 shows the plasma electron density profiles on axis for all three cases as obtained from the PIC simulations $\approx 1$ ps before the electron beam driven blowout hits the plasma torch at $z=3.0 \mathrm{~mm}$. Cases (i) and (ii) are combined in Fig. 2(a), where the electron driver is depicted with the green line at $z \approx 2.65 \mathrm{~mm}$. The electric field concurrent with
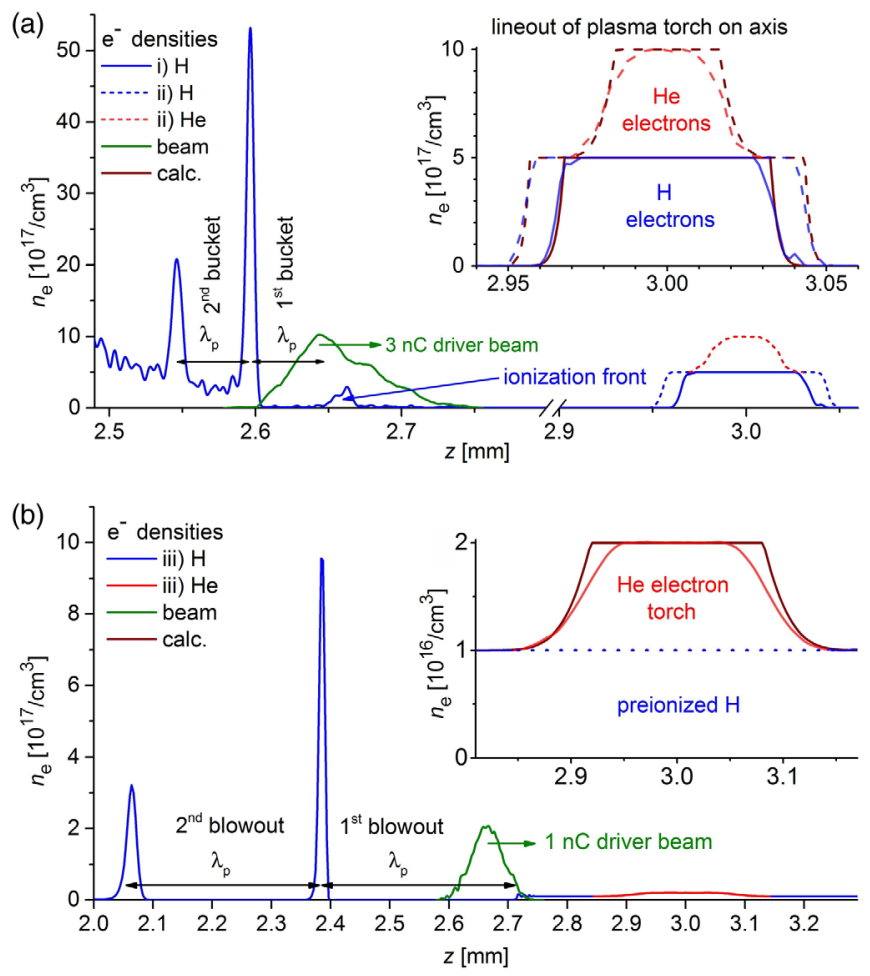

FIG. 2. On-axis density lineouts for cases (i) and (ii), where hydrogen and helium are neutral (a), and (iii), where hydrogen is preionized (b). The electron bunch driver (green) propagates to the right, and the torch is produced by a laser pulse with $a_{0}=$ 0.015 in case (i) and $a_{0}=0.025$ in case (ii) and (iii). The zoomed insets show the plasma torch profile obtained via numeroanalytical ADK calculations (brown) compared to densities obtained in the simulation (blue/red). 
the driver beam exceeds the hydrogen ionization threshold not before close to the driver bunch maximum, which then gives rise to the hydrogen ionization front and electron density peak (blue solid line). These hydrogen electrons are expelled from axis, so that their on-axis density vanishes. This constitutes the first plasma wave bucket, and after the plasma wavelength $\lambda_{\mathrm{p}}=2 \pi c \sqrt{\epsilon_{0} m_{e} / n_{e} e^{2}} \approx 47 \mu \mathrm{m}$ (black double-headed arrows), the electron density bounces up again at $z \approx 2.6 \mathrm{~mm}$ dramatically due to overshooting plasma wave electrons with a peak density on axis of $n_{\mathrm{e}, \mathrm{H}} \approx 53 \times 10^{17} \mathrm{~cm}^{-3}$. This is being followed by a second wave bucket with reduced peak density on axis at the plasma wave vertex.

The combination of laser waist $w_{0}$ and $a_{0}$ have been chosen such that the torch width $L \approx \lambda_{\mathrm{p}}$, in order to allow for the plasma wave to interact at increased density at least over one plasma wavelength. On the other hand, a compact torch allows for rapid density transition, and it is known that the downramp length $l$ should be shorter than the plasma skin depth $k_{\mathrm{p}}^{-1}=c / \omega_{\mathrm{p}}$ [42] for effective injection. This criterion is fulfilled in each case. In case (i) $w_{0}=$ $40 \mu \mathrm{m}$ and $a_{0}=0.015$, which leads to a total torch width $L_{\mathrm{i}} \approx 80 \mu \mathrm{m}$ as estimated by numero-analytical AmmosovDelone-Krainov (ADK) tunnel ionization yields (brown line) [43] and as confirmed by the PIC simulations. The trapezoidal hydrogen electron density profile (blue line) of the plasma torch is the result of complete ionization, and the density ramp is very steep, with the hydrogen electron density ramping up from zero to $n_{\mathrm{e}, \mathrm{H}}=5 \times 10^{17} \mathrm{~cm}^{-3}$ on a length of $l_{\mathrm{i}} \approx 10 \mu \mathrm{m}$. In case (ii), the ramp with $l_{\mathrm{ii}} \approx 35 \mu \mathrm{m}$ additionally comprises helium electrons (red dashed line), which leads to a double-trapezoidal profile of $n_{\mathrm{e}, \mathrm{H}}+$ $n_{\mathrm{e}, \mathrm{He}}=10 \times 10^{17} \mathrm{~cm}^{-3}$ maximum density, and total plasma torch width of $L_{\mathrm{ii}} \approx 110 \mu \mathrm{m}$. This was triggered by a stronger laser pulse of $a_{0}=0.025$ at the same waist size.

In case (iii), as shown in Fig. 2(b), the situation is substantially different due to the fact, that preionized hydrogen is used. Therefore the wakefield is much stronger and would at the same hydrogen density ionize and even trap helium electrons $[24,29,35]$, which is unwanted here. Because of that, the hydrogen/helium densities are chosen an order of magnitude lower, increasing $\lambda_{\mathrm{p}, \mathrm{H}} \approx 334 \mu \mathrm{m}$, and the driver beam charge $Q$ was reduced from 3 to $1 \mathrm{nC}$. Additionally, in order to make the torch extend over approximately the plasma wavelength, the laser waist size has been increased to $w_{0}=200 \mu \mathrm{m}$ with $a_{0}=0.025$. This leads to a total plasma torch width $L_{\mathrm{iii}} \approx 300 \mu \mathrm{m}$, and density ramp length $l_{\mathrm{iii}} \approx 80 \mu \mathrm{m}$.

Figure 3 shows snapshots of the interaction for case (i), using temporally consecutive plots of the longitudinal electric field $E_{z}(\mathrm{a}, \mathrm{d})$, the total electron density $n_{e}(\mathrm{~b})$, and the corresponding potential $\Phi(\mathrm{c})$, overlayed by drive beam (green), and hydrogen-electrons (blue). In addition an

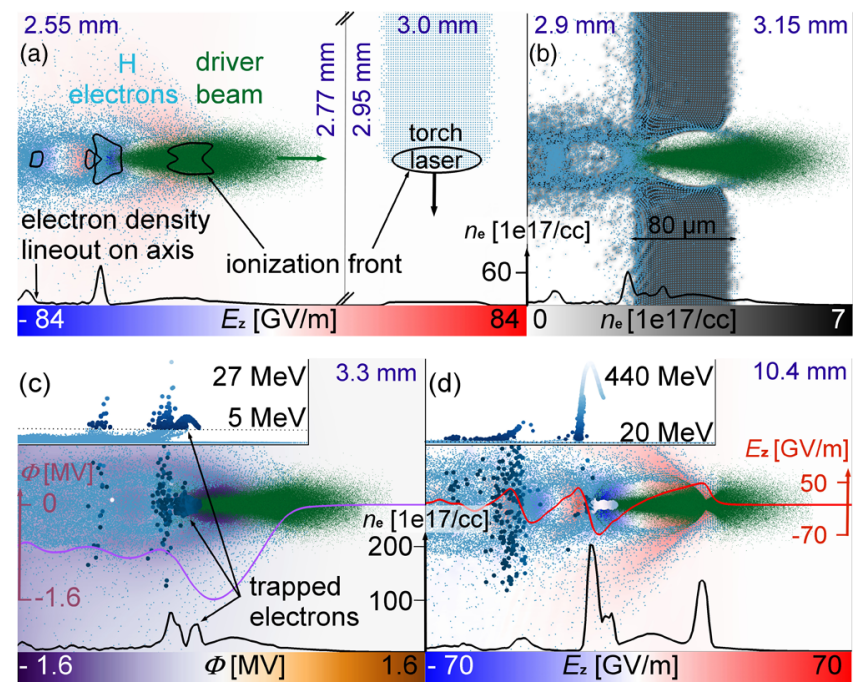

FIG. 3. Optical plasma torch injection for case (i). In (a) the plasma torch is generated in the path of the drive beam, and in (b) the torch is crossed, leading to blowout amplification and injection. The injected hydrogen electrons are shown in (c) and (d) after $z \approx 10.4 \mathrm{~mm}$ of acceleration, where maximum energies of $E \approx 440 \mathrm{MeV}$ are reached.

electron density lineout on axis (black line) is situated at the bottom of each snapshot (similar to Fig. 2). In Fig. 3(a), the bunch has not yet reached the hydrogen torch. The longitudinal electric field is given by a $2 \mathrm{~d}$ slice plot (red/blue colorbar) with accelerating field values down to $\approx-84 \mathrm{GV} / \mathrm{m}$, while the electron bunch self field exceeds the hydrogen ionization threshold only close to its density maximum.

The ionization front, here defined as where $W_{\mathrm{ADK}}>$ $0.1 \mathrm{fs}^{-1}$, is indicated (black isoline). In Fig. 3(b), the driver bunch is entering the torch, which means that the driver locally interacts with preionized hydrogen and for the length of the plasma torch, the hydrogen electrons are expelled much earlier than in the self-ionized case. This results in a pronounced change of the plasma wave shape: the head of the drive bunch can already contribute to the generation of the blowout, which is therefore locally considerably amplified. The beginning of the plasma blowout jumps forward from approximately the center to the very front of the driver bunch. When leaving the plasma torch region, the blowout is again shifted backwards rapidly, which leads to controlled injection. It is interesting to note that the plasma density after passage of the drive beam is the same outside and inside the plasma torch in contrast to state-of-the-art downramp injection. The strong plasma wave blowout can close before the end of the torch is reached as $L_{\mathrm{i}} \approx 80 \mu \mathrm{m}>\lambda_{\mathrm{p}} \approx 47 \mu \mathrm{m}$. The effectiveness of the plasma region for injection is seen in snapshot 3(c) at $200 \mu \mathrm{m}$ after the plasma torch. Hydrogen electrons are injected as the plasma wave is suddenly retarded and the hydrogen expulsion is shifting back to near the drive beam density maximum. 
The inset visualizes the longitudinal phase space of injected and trapped electrons, with electrons $>5 \mathrm{MeV}$ being shown as bigger blue spheres. Snapshot 3(d) illustrates the fields and particles after $z \approx 10.4 \mathrm{~mm}$ of acceleration. A considerable amount of hydrogen electrons $Q_{\mathrm{i}} \approx 0.24 \mathrm{nC}$ (peak current of $I_{\mathrm{i}} \approx 9.4 \mathrm{kA}$ ) has been trapped and is accelerated with total energy spread of $\approx 15.3 \%$, maximum energies of $E \approx 440 \mathrm{MeV}$, and a normalized emittance of $\epsilon_{\mathrm{n}} \approx 2.0 \times 10^{-6} \mathrm{~m} \mathrm{rad}$.

A main advantage of case (ii), where the torch laser energy is increased from $0.8 \mathrm{~mJ}$ of case (i), to $2.3 \mathrm{~mJ}$ to reach an intensity of $a_{0}=0.025$ required to ionize helium, is that the injected charge can be regulated via the helium gas density. For example, at $n_{\mathrm{He}}=n_{\mathrm{H}}=5 \times 10^{17} \mathrm{~cm}^{-3}$ the plasma torch density is doubled and the additional helium leads to a nearly doubled witness bunch charge $Q_{\mathrm{ii}}=$ $0.53 \mathrm{nC} \approx 2 Q_{\mathrm{i}}$ when compared to case (i). While other witness bunch main characteristics such as peak energy $(E \approx 498 \mathrm{MeV})$ and normalized emittance $\left(\epsilon_{\mathrm{n}} \approx 2.4 \times\right.$ $10^{-6} \mathrm{~m} \mathrm{rad}$ ) are hardly affected by the additional charge, the total energy spread increases to $35.5 \%$. A series of simulations confirms, that there is a linear scaling (within beam loading limits) between the plasma torch density and the injected charge-which makes it possible to steer the injected charge simply by tuning the helium gas density.

In case (iii) — where hydrogen is completely preionizedan entirely different picture arises. At similar densities and drive beam currents as in (i) and (ii), continuous injection due to the strong wakefields would occur [24,29,35]. Therefore, more than an order of magnitude lower gas densities $n_{\mathrm{He}}=n_{\mathrm{H}}=1 \times 10^{16} \mathrm{~cm}^{-3}$ are used, and in addition the drive beam charge is reduced to $1 \mathrm{nC}$. This relaxes the requirements on the driving electron beam, and decreases the electric field at the "hot spots" for example at the blowout vertex, and thus avoids unwanted ionization and potential dark current generation.

For the sake of comparability, here we do not adjust the drive bunch duration but operate substantially below the optimal case $k_{\mathrm{p}} \sigma_{\mathrm{z}} \approx 0.5<\sqrt{2}$, leaving room for further relaxing the drive bunch requirements by increasing $\sigma_{\mathrm{z}}$. On the other hand, because of the much longer $\lambda_{\mathrm{p}} \approx 334 \mu \mathrm{m}$, the torch has to be substantially broader to have a significant impact on the wake. Hence the torch laser has a local spot size of $w_{0}=200 \mu \mathrm{m}$, while keeping $a_{0}=0.025$ as in the previous case.

The scenario is illustrated in Fig. 4, using a similar visualization as in Fig. 3. Figure 4(a) shows that preionized hydrogen leads to pronounced blowout formation, with peak accelerating electric fields on axis of $E_{z}=$ $-13 \mathrm{GV} / \mathrm{m}$ (red lineout) before the helium-based plasma torch is reached (compare Fig. 3). When entering the torch, the plasma density ramps up to an electron density value of $n_{\mathrm{e}, \mathrm{H}}+n_{\mathrm{e}, \mathrm{He}}=2 \times 10^{16} \mathrm{~cm}^{-3}$, which leads to a contraction of the blowout cavity to $\lambda_{\mathrm{p}} \approx 236 \mu \mathrm{m}$. The blowout cavity sizes before and amid the plasma torch are indicated by

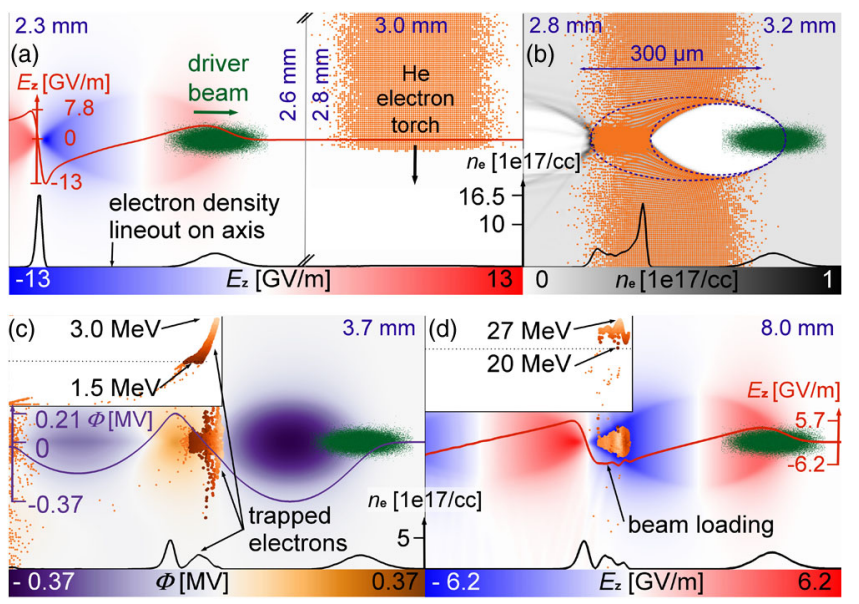

FIG. 4. Plasma torch injection in case (iii). In preionized hydrogen, at gas densities of $n_{\mathrm{He}}=n_{\mathrm{H}}=1 \times 10^{16} \mathrm{~cm}^{-3}$ a pronounced blowout is generated (a) and the tunable helium torch (b) downramp leads to dark-current free witness bunch formation (c) and allows for massive charge at substantially beam-loaded levels (d).

dashed ellipses in Fig. 4(b). The downramp at the end of the plasma torch is very effective for injection and trapping of plasma electrons, in fact much more effective than in cases (i) and (ii).

Figure 4(c) shows the injected charge immediately after the downramp, using the electrostatic wake potential $\Phi$ as a color plot, with an on-axis lineout (purple line). At this helium density level, the injected charge $Q_{\text {iii }}$ (both hydrogen and helium electrons) is massive and on the same order of magnitude as the driver beam charge. This is best seen from the black on-axis density lineout. The first peak of the double peak represents injected charge and the second peak is produced by overshooting hydrogen plasma electrons. The inset shows the longitudinal phase space, with the dashed line indicating the $1.5 \mathrm{MeV}$ energy level. One can see that the generated electron witness bunch is dark current free, thanks to the nature of the localized injection process. Not all of the depicted electron macroparticles are trapped, but there is a fraction with high transverse momenta which is quickly lost to the plasma. This cleanses the bunch and reduces its emittance and energy spread.

In Fig. 4(d) the produced bunch has reached the 20-27 MeV energy level after $z \approx 8.0 \mathrm{~mm}$, at a reduced maximum accelerating field of $E_{z} \approx-6.2 \mathrm{GV} / \mathrm{m}$, compared to snapshot (a). The trapped charge amounts $Q_{\mathrm{iii}} \approx$ $0.26 \mathrm{nC}$ and leads to massive beam loading, as evidenced by the reduced peak electric field. The energy spread amounts to $6.4 \%$ - substantially smaller than in cases (i) and (ii) - and the normalized transverse emittance of $\approx 2.6 \times 10^{-6} \mathrm{~m} \mathrm{rad}$. As in case (ii), the tunability of the helium density is a powerful tool to adjust the injected charge and other bunch characteristics.

In conclusion, it shall be emphasized that hydrogen preionization leads to the best results and also allows for 
highest tunability. However, as expected and as observed in further sets of simulations, almost any distortion of the wakefield loads some charge into the blowout, even in asymmetric cases when the torch is somewhat off axis, but broad enough to be in the blowout's path. The charge of the injected electrons can be tuned via the density of the torch (tuning the HIT medium pressure) up to beam loading level [compare Figs. 4(a) and (d)]. The trapping position and bunch length can be controlled by the relative height of the torch, which is given by the gas mixture ratio $n_{\mathrm{e}, \mathrm{H}} / n_{\mathrm{e}, \mathrm{He}}$. Further optimization can be achieved, and demands on laser requirements can be substantially relaxed by operating at increased helium gas densities (which is experimentally straightforward) while decreasing the laser spot size, and by using different types of lasers and wavelengths.

We have presented a fundamentally new, flexible, fully optically-steered method to generate tunable plasma torch electron density transitions. Density transitions are so far produced via hydrodynamic mechanisms in a variety of schemes both in LWFA and PWFA, which comes with severe limitations as they are based on kinetic movement of gas molecules with velocities limited to a few $1000 \mathrm{~m} / \mathrm{s}$ and furthermore require proximity of more or less complex mechanical components (e.g., razor blades, gas nozzles, differential pumping stages, etc.) close to the interaction point. In contrast, optical shaping via plasma torch generation happens with $c \approx 3 \times 10^{8} \mathrm{~m} / \mathrm{s}$ and does not require any movement of gas molecules.

The produced plasma electron density profile is a direct signature of the applied laser intensity profile, and therefore allows us to produce very steep, tailored density ramps on fs time scales. By tuning the laser energy, wavelength, focus size, and position, the density transition can be tuned in a wide parameter range. Using axicon(s) and/or higher laser modes, multifoci, line foci, etc. to shape the electron density profile would also be possible and would extend the accessible parameter range further.

Here we have used the method to produce sharp electron density transitions in front of the electron beam-driven plasma wave to trigger injection. Other applications would be plasma electron density shaping at the exit and entrance of a plasma electron accelerator stage in order to preserve bunch qualities, and ultrafast bunch kickers by production of asymmetric plasma density profiles as demonstrated in [39]. The latter was realized in PIC simulations (not shown here) by simply changing the angle between torch laser and electron bunch propagation axis to $5.24 \mathrm{mrad}$, so a $E=250 \mathrm{MeV}, Q=120 \mathrm{pC}$ bunch was deflected by an angle of $\Theta \approx 1.7 \mathrm{mrad}$ exiting a helium torch of $n_{\mathrm{He}}=$ $1 \times 10^{18} \mathrm{~cm}^{-3}$ density. These applications are in particular relevant to PWFA, where low ionization thresholds and multiple gas types can be used. Here the gas density of different gas species can be uniform over the whole acceleration stage-whereas the production of multicomponent mixtures with (independently) differing, localized gas density profiles $[29,38]$ is experimentally challenging.

That said, the method can also have applicability to LWFA if using higher laser intensities for the torch laser when compared to the drive laser similar as in [30]. Finally, the proposed method does open up a path to higher repetition rates and higher efficiency when compared to hydrodynamic solutions.

This work was supported by DFG, STFC 4070022104, DOE DE-SC0009533, DE-FG02-07ER46272, DE-FG0392ER40693, by ONR N00014-06-1-0925 and Helmholtz VH-VI-503. We acknowledge the assistance of the VSim development team. This research used computational resources of the National Energy Research Scientific Computing Center, which is supported by DOE DE-AC02-05CH11231, and of JUROPA, and of HLRN. D. A. J. acknowledges support of the UK EPSRC (EP/J018171/1) and the ECs 7th Framework Programme (LASERLAB-EUROPE No. 284464, EUCARD-2 Project No. 312453) and the Extreme Light Infrastructure (ELI).

[1] T. Tajima and J. M. Dawson, Phys. Rev. Lett. 43, 267 (1979).

[2] A. Pukhov and J. Meyer-ter Vehn, Appl. Phys. B 74, 355 (2002).

[3] S. P. D. Mangles, C. D. Murphy, Z. Najmudin, A. G. R. Thomas, J. L. Collier, A. E. Dangor, E. J. Divall, P. S. Foster, J. G. Gallacher, C. J. Hooker, D. A. Jaroszynski, A. J. Langley, W. B. Mori, P. A. Norreys, F. S. Tsung, R. Viskup, B. R. Walton, and K. Krushelnick, Nature (London) 431, 535 (2004).

[4] C. G. R. Geddes, C. Toth, J. v. Tilborg, E. Esarey, C. B. Schroeder, D. Bruhwilder, C. Nieter, J. Cary, and W. P. Leemans, Nature (London) 431, 538 (2004).

[5] J. Faure, Y. Glinec, A. Pukhov, S. Kiselev, S. Gordienko, E. Lefebvre, J. P. Rousseau, F. Burgy, and V. Malka, Nature (London) 431, 541 (2004).

[6] W. P. Leemans, B. Nagler, A. J. Gonsalves, Cs. Tóth, K. Nakamura, C. G. R. Geddes, E. Esarey, C. B. Schroeder, and S. M. Hooker, Nat. Phys. 2, 696 (2006).

[7] X. Wang et al., Nat. Commun. 4, 1988 (2013).

[8] W. P. Leemans, A. J. Gonsalves, H.-S. Mao, K. Nakamura, C. Benedetti, C. B. Schroeder, C. Toth, J. Daniels, D. E. Mittelberger, S. S. Bulanov, J.-L. Vay, C. G. R. Geddes, and E. Esarey, Phys. Rev. Lett. 113, 245002 (2014).

[9] P. Chen, J. M. Dawson, R. W. Huff, and T. Katsouleas, Phys. Rev. Lett. 54, 693 (1985).

[10] J. B. Rosenzweig, D. B. Cline, B. Cole, H. Figueroa, W. Gai, R. Konecny, J. Norem, P. Schoessow, and J. Simpson, Phys. Rev. Lett. 61, 98 (1988).

[11] I. Blumenfeld, C. E. Clayton, F.-J. Decker, M. J. Hogan, C. Huang, R. Ischebeck, R. Iverson, C. Joshi, T. Katsouleas, N. Kirby, W. Lu, K. A. Marsh, W. B. Mori, P. Muggli, E. Oz, R. H. Siemann, D. Walz, and M. Zhou, Nature (London) 445, 741 (2007). 
[12] N. Vafaei-Najafabadi et al., Phys. Rev. Lett. 112, 025001 (2014).

[13] M. Litos et al., Nature (London) 515, 92 (2014).

[14] S. Bulanov, N. Naumova, F. Pegoraro, and J. Sakai, Phys. Rev. E 58, R5257 (1998).

[15] H. Suk, N. Barov, J. B. Rosenzweig, and E. Esarey, Phys. Rev. Lett. 86, 1011 (2001).

[16] C. G. R. Geddes, K. Nakamura, G. R. Plateau, C. Toth, E. Cormier-Michel, E. Esarey, C. B. Schroeder, J. R. Cary, and W. P. Leemans, Phys. Rev. Lett. 100, 215004 (2008).

[17] J. Faure, C. Rechatin, O. Lundh, L. Ammoura, and V. Malka, Phys. Plasmas 17, 083107 (2010).

[18] K. Schmid, A. Buck, C. M. S. Sears, J. M. Mikhailova, R. Tautz, D. Herrmann, M. Geissler, F. Krausz, and L. Veisz, Phys. Rev. ST Accel. Beams 13, 091301 (2010).

[19] A. J. Gonsalves, K. Nakamura, C. Lin, D. Panasenko, S. Shiraishi, T. Sokollik, C. Benedetti, C. B. Schroeder, C. G. R. Geddes, J. van Tilborg, J. Osterhoff, E. Esarey, C. Toth, and W. P. Leemans, Nat. Phys. 7, 862 (2011).

[20] P. Brijesh, C. Thaury, K. T. Phuoc, S. Corde, G. Lambert, V. Malka, S. P. D. Mangles, M. Bloom, and S. Kneip, Phys. Plasmas 19, 063104 (2012).

[21] D. Umstadter, J.-K. Kim, and E. Dodd, U.S. Patent Ser. No. 5,789,876 (1995).

[22] M. Chen, Z.-M. Sheng, Y.-Y. Ma, and J. Zhang, J. Appl. Phys. 99, 056109 (2006).

[23] D. Umstadter, J. K. Kim, and E. Dodd, Phys. Rev. Lett. 76, 2073 (1996).

[24] E. Oz et al., Phys. Rev. Lett. 98, 084801 (2007).

[25] C. McGuffey, A. G. R. Thomas, W. Schumaker, T. Matsuoka, V. Chvykov, F. J. Dollar, G. Kalintchenko, V. Yanovsky, A. Maksimchuk, K. Krushelnick, V. Y. Bychenkov, I. V. Glazyrin, and A. V. Karpeev, Phys. Rev. Lett. 104, 025004 (2010).

[26] A. Pak, K. A. Marsh, S. F. Martins, W. Lu, W. B. Mori, and C. Joshi, Phys. Rev. Lett. 104, 025003 (2010).

[27] C. E. Clayton, J. E. Ralph, F. Albert, R. A. Fonseca, S. H. Glenzer, C. Joshi, W. Lu, K. A. Marsh, S. F. Martins, W. B. Mori, A. Pak, F. S. Tsung, B. B. Pollock, J. S. Ross, L. O. Silva, and D. H. Froula, Phys. Rev. Lett. 105, 105003 (2010).

[28] M. Chen, E. Esarey, C. B. Schroeder, C. G. R. Geddes, and W. P. Leemans, Phys. Plasmas 19, 033101 (2012).
[29] A. Martinez de la Ossa, J. Grebenyuk, T. Mehrling, L. Schaper, and J. Osterhoff, Phys. Rev. Lett. 111, 245003 (2013).

[30] N. Bourgeois, J. Cowley, and S. M. Hooker, Phys. Rev. Lett. 111, 155004 (2013).

[31] B. Hidding, G. Pretzler, D. Bruhwiler, and J. Rosenzweig, German Patent No. DE 102011104858.1 (2011); US/PCT Patent Ser. No. PCT/US12/043002 (2011).

[32] B. Hidding, G. Pretzler, J. B. Rosenzweig, T. Koenigstein, D. Schiller, and D. L. Bruhwiler, Phys. Rev. Lett. 108, 035001 (2012).

[33] B. Hidding, J. B. Rosenzweig, Y. Xi, B. O'Shea, G. Andonian, D. Schiller, S. Barber, O. Williams, G. Pretzler, T. Königstein, F. Kleeschulte, M. J. Hogan, M. Litos, S. Corde, W. W. White, P. Muggli, D. L. Bruhwiler, and K. Lotov, AIP Conf. Proc. 1507, 570 (2012).

[34] Y. Xi, B. Hidding, D. Bruhwiler, G. Pretzler, and J. B. Rosenzweig, Phys. Rev. ST Accel. Beams 16, 031303 (2013).

[35] F. Li, J. F. Hua, X. L. Xu, C. J. Zhang, L. X. Yan, Y. C. Du, W. H. Huang, H. B. Chen, C. X. Tang, W. Lu, C. Joshi, W. B. Mori, and Y. Q. Gu, Phys. Rev. Lett. 111, 015003 (2013).

[36] X. L. Xu et al., Phys. Rev. Lett. 112, 035003 (2014).

[37] B. Hidding et al., arXiv:1403.1109.

[38] L.-L. Yu, E. Esarey, C. B. Schroeder, J.-L. Vay, C. Benedetti, C. G. R. Geddes, M. Chen, and W. P. Leemans, Phys. Rev. Lett. 112, 125001 (2014).

[39] P. Muggli, S. Lee, T. Katsouleas, R. Assmann, F. J. Decker, M. J. Hogan, R. Iverson, P. Raimondi, R. H. Siemann, D. Walz, B. Blue, C. E. Clayton, E. Dodd, R. A. Fonseca, R. Hemker, C. Joshi, K. A. Marsh, W. B. Mori, and S. Wang, Phys. Rev. ST Accel. Beams 4, 091301 (2001).

[40] T.-Y. Chien, C.-L. Chang, C.-H. Lee, J.-Y. Lin, J. Wang, and S.-Y. Chen, Phys. Rev. Lett. 94, 115003 (2005).

[41] C. Nieter and J. R. Cary, J. Comput. Phys. 196, 448 (2004).

[42] H. Suk, N. Barov, J. B. Rosenzweig, and E. Esarey, Phys. Rev. Lett. 86, 1011 (2001).

[43] D. L. Bruhwiler, D. A. Dimitrov, J. R. Cary, E. Esarey, W. Leemans, and R. E. Giacone, Phys. Plasmas 10, 2022 (2003). 